\title{
Métodos computarizados y algunas de sus aplicaciones al estudio de la flora de México ${ }^{1}$
}

\author{
Nancy P. Moreno ${ }^{2}$ y Robert Allkin ${ }^{3}$
}

\begin{abstract}
RESUMEN. El uso de las bases de datos computarizadas puede ser una alternativa viable para apoyar el estudio de la flora de México; sin embargo, hay pocos trabajos escritos en español sobre el tema. Aquí se pretende dar una introducción al área, presentando los tres tipos de bases de datos aplicables en general a estudios florísticos: 1) bases curatoriales, con datos de etiquetas de ejemplares de herbario; 2) bases bibliográficas, con datos acerca de la literatura taxonómica, y 3 ) bases descriptivas, las cuales contienen descripciones de los organismos mismos. Estas últimas facilitan la producción de medios auxiliares para la identificación, la publicación de descripciones taxonómicas o el acceso a cualquier subconjunto de la información almacenada en la computadora.
\end{abstract}

ABSTRACT. The use of computerized databases could be a viable alternative in the study of the flora of Mexico. However, very few articles have been published in Spanish about this area of research. This contribution is intended to provide an introduction to the field, with commentaries on the three types of databases most applicable to floristic studies. These are: 1) curatorial databases, concerned with the information from specimen labels; 2) bibliographic databases, containing reference to taxonomic literature, and 3 ) descriptive databases that permit the automatic production of identification aids of taxonomic descriptions derived from previously stored information.

Ahora más que nunca se reconoce la necesidad de estudiar la flora de México. Los ecosistemas naturales están sujetos cada día más a la influencia directa e indirecta de las demandas de una población creciente (Gómez-Pompa et al., 1972; Prance, 1977). La alteración irreversible de algunos de estos ecosistemas hace imperativa la tarea del taxónomo en documentar su composición florística. De la misma manera, sólo por medio de estudios taxonómicos y florísticos detallados, entre otros tipos de trabajos, será posible demostrar el valor científico y genético de estos sitios.

La creación del Consejo Nacional para la Flora de México demuestra la aceptación

${ }^{1}$ Trabajo parcialmente subvencionado por el Consejo Nacional de Ciencia y Tecnología; también se reconoce al apoyo brindado por el Consejo Británico.

${ }^{2}$ Instituto Nacional de Investigaciones sobre Recursos Bióticos, Apdo. Postal 63, Jalapa, Veracruz.

${ }^{3}$ Biology Dept. University of Southampton, England S09 5NM.

Moreno NP, Allkin R. 1988. Métodos computarizados y algunas de sus aplicaciones al estudio de la flora de México. Boletín de la Sociedad Botánica de México 48: 65-73. 
de este compromiso por parte de la comunidad taxonómica nacional. Sin embargo, no se ha llegado a un consenso sobre la mejor manera de llevar a cabo una empresa de tal magnitud.

Sin duda, será necesario integrar grandes cantidades de información proveniente tanto de la bibliografía ya disponible como de ejemplares botánicos. Esta información deberá estar a la disposición de los diversos colaboradores para permitir el aprovechamiento mutuo de los diferentes avances y para facilitar la coordinación misma de la investigación. Aunque esto puede llevarse a cabo mediante métodos tradicionales para el manejo y procesamiento de información, vale la pena considerar los medios electrónicos como otra opción viable para el mismo fin.

Esto no significa que deba optarse por una flora completamente "automatizada". Como se vio en el proyecto inconcluso para la flora de Norteamérica (Krauss, 1973), manejar cada uno de los diferentes tipos de información requeridos para la integración de una flora por medios computarizados presenta varios problemas de coordinación y de operatividad (Shetler, 1974). Sin embargo, es factible manejar una gran parte de los datos rutinarios asociados con este tipo de trabajo en bases de datos almacenadas en la computadora y, en el proceso, aliviar la carga de trabajos tediosos o rutinarios de los taxónomos. El uso de este tipo de metodología puede ofrecer ventajas considerables para los usuarios, ya que permite el acceso flexible a la información y la corrección y adición de datos en cualquier momento.

De hecho, existe ya un número creciente de aplicaciones de sistemas de bases de datos a la taxonomía y a la florística. Los avances tecnológicos acoplados con los adelantos en programación permiten cada día mayor facilidad en la captura, almacenamiento y recuperación de datos. Desafortunadamente, casi toda la literatura relacionada con este tema está escrita en inglés, lo cual dificulta su manejo por algunos de los biólogos que podrían estar interesados en el método. Aquí se pretende dar una breve introducción general al tema y se sugieren algunos trabajos para mayor información.

Por "base de datos" se entiende una colección de datos relacionados, almacenados juntos. A pesar de que ésta podría referirse a cualquier conjunto de información, por lo general se trata de información guardada en un formato especial dentro de una computadora. Los diferente programas diseñados para la captura, manipulación y recuperación de los datos y que actúan en forma intermediaria entre el usuario y la base de datos, constituyen el sistema de procesamiento o de manejo de datos. El término "banco de datos" es menos específico y con frecuencia se utiliza como sinónimo de base de datos. Aunque cada sistema tiene sus particularidades, en general nos permite guardar información específica, por ejemplo, nombres, direcciones y especialidades de cada uno de los botánicos en México, y después consultarla (toda o en parte) y reproducirla en cualquier formato. En el caso del ejemplo, sería factible (entre muchas otras posibilidades) generar listas separadas de los practicantes de cada especialidad sin tener que hacer cambios manuales en los datos.

Crovello (1967) establece tres tipos de información florística que pueden ser manejados por medios electrónicos: 1) la relacionada con las etiquetas de ejemplares de herbario o el manejo de los mismos (base de datos curatoriales); 2) la proveniente del ejemplar mismo, o sea los caracteres taxonómicos de la planta (base de datos florísticos, monográficos o descriptivos), y 3) cualquier información externa al ejemplar, como serían listados bibliográficos, etcétera. 
En las siguientes secciones se describen algunos de los diferentes tipos de información que pueden ser manejados con bases de datos pertenecientes a la florística.

\section{BASES DE DATOS CURATORIALES}

Los sistemas diseñados para procesar información relacionada con las etiquetas de ejemplares de herbario o la contabilidad interna de los mismos se consideran curatoriales. En otras palabras, ayudan en mayor o menor grado al curador o encargado del herbario para realizar funciones. Dentro de esta categoría se incluyen los sistemas que almacenan y recuperan información proveniente de etiquetas, los que imprimen etiquetas, los que contabilizan los ejemplares prestados, los recibidos en préstamo, los enviados para identificación, etc., así como cualquier base de datos complementario que contenga nombres científicos, abreviaturas o localidades, por ejemplo.

Los primeros sistemas de este tipo se originaron hace aproximadamente 15 años (v.g. Crovello, 1972; MacDonald, 1966; Scheinvar et al., 1967; Soper y Perring, 1967). De acuerdo con el equipo disponible la información se capturaba en cinta o en tarjetas perforadas, para transferirse después a cinta magnética. Frecuentemente las opciones para el uso de los datos eran limitadas y el procesamiento de la información era lento. Actualmente es posible obtener mayor flexibilidad en cuanto al manejo de los datos. Casi todos los sistemas en uso ahora permiten la captura de información directamente en una terminal con pantalla. Esto se aplica tanto en el caso de las computadoras grandes como en las numerosas microcomputadoras ahora disponibles en el mercado.

Herbarios tan diversos como el Herbario Nacional en Pretoria (PRE) (Gibbs Russell y Consalves, 1984), el Herbario Nacional de Colombia (COL) (Forero y Pereira, 1976), el Herbario Edward Lee Green en la Universidad de Notre Dame (ND-G) (Crovello, 1972), el Herbario del Instituto Nacional de Investigaciones sobre Recursos Bióticos (XAL) (Gómez-Pompa et al., 1985), entre otros, cuentan con sistemas operativos desde hace algunos años. Otros herbarios como el del Jardín Botánico de Ginebra (G) (Mascherpa y Bocquet, 1984) y el Herbario Nacional de México (MEXU), se encuentran en proceso de poner en práctica diferentes tipos de sistemas curatoriales. Según las estimaciones de Pankhurst (1984) hay nueve bases de datos de este tipo en el mundo con más de 50000 registros, excluyendo jardines botánicos y arboreta.

Aunque cada banco de datos tiene algunas características particulares, generalmente consta de los datos provenientes de los ejemplares en el herbario, representados en un formato estandarizado, con la posibilidad de recuperarlos en diferentes presentaciones. El manejo es generalmente directo, mediante una terminal con pantalla que permite la interacción del usuario con la base de datos. Los complejos sistemas operativos reales son invisibles para el usuario, quien no necesita tener conocimiento especializado de computación para utilizar el sistema. Algunos de los nuevos sistemas comerciales para bases de datos en microcomputadora son tan sencillos que tampoco se requiere experiencia en computación para adaptarlos a aplicaciones particulares.

\section{BASES DE DATOS BIBLIOGRÁFICOS Y NOMENCLATURALES}

El manejo de información bibliográfica es una de las áreas de mayor desarrollo en cuanto a bases de datos científicos. Los servicios internacionales de búsqueda de literatu- 
ra (v.gr. Orbit [Systems Development Corporation, 1975] o Dialog [Lockheed Information Systems, sin fecha]) son ampliamente conocidas y de utilidad indiscutible. También existen varios bancos computarizados de bibliografía pertenecientes a proyectos específicos, donde se almacenan las citas relevantes al trabajo en desarrollo (varios ejemplos en Crovello y MacDonald, 1970); o relacionados con temas especiales, por ejemplo Biosis, mantenido por Biosciences Information Service, el mismo servicio que produce Biological Abstracts (Abbot et al., 1985).

Generalmente, las bases de este tipo contienen información comparable a la que se encuentra en tarjetas de registro bibliotecario, como autor, título, año, descripción corta del contenido, clasificación, etc. Por lo regular también incluyen una o varias "palabras clave" relacionadas con los temas de la publicación y que facilitan la búsqueda de citas de algún tema en particular.

La aplicación de este tipo de base de datos para estudios florísticos en México es obvia. Mediante uno o varios bancos centralizados, se podría facilitar el acceso por botánicos de diferentes instituciones a los recursos bibliográficos localizados en diversas partes del país, eliminando así la necesidad de llevar a cabo viajes costosos o la duplicación de recursos.

También es de importancia para los estudios sistemáticos en México el almacenamiento de información nomeclatural en bases de datos. En esta área, los zoólogos se encuentran mucho más avanzados que los taxónomos botánicos. Para el Zoological Record, equivalente a los índices Kewensis y Gray, se mantienen las citas de descripciones nuevas en una base de datos desde hace varios años, la cual facilita el manejo y la presentación de la información, además de promover la cooperación y colaboración a nivel mundial. Se reporta (Lucas, 1984) que se planea tener el Index Kewensis disponible en esa forma dentro de algunos años.

En el Proyecto Flora de Veracruz, desde su comienzo, se ha mantenido una lista de nombres correctos y sinónimos de las especies reportadas para el estado (Olvera Fonseca y Gómez-Pompa, 1973) la cual funciona como referencia central para los demás componentes del sistema.

El Proyecto Vicieae (Vicieae Database Project) (Bisby et al., 1983) maneja bases de datos nomenclaturales y bibliográficos dirigidos especialmente a esa tribu de leguminosas. Éstas tienen dos funciones dentro del proyecto: 1) como servicio de información sobre la nomeclatura del grupo y 2) como parte fundamental para los otros componentes del conjunto de bases de datos sobre el grupo.

Para Europa, el Sistema Europeo para la Documentación Taxonómica (Heywood et al., 1984) está incorporando la información sistemática, geográfica, ecológica y cromosómica proveniente inicialmente de la Flora Europea en una base de datos. El propósito es proveer un sistema de información florística, biosistemática y taxonómica acerca de las plantas vasculares de Europa, que se puede consultar directamente emplearse para producir catálogos revisados de sinónimos y otros tipos de información biosistemática.

\section{BASES DE DATOS DESCRIPTIVOS}

Un área relativamente nueva es la aplicación de métodos computarizados para la descripción de organismos. A menudo se hace referencia a este tipo de base de datos 
como florística o monográfica, ya que cumple con los mismos propósitos de una flora o una monografía, según su diseño. En otras palabras, reúne información acerca de las características de taxa, nomenclatura, distribución geográfica, etc. y provee medios para su identificación.

En sistemas de este tipo generalmente se almacenan descripciones en un formato especial dentro de la computadora. Cada descripción consiste en una lista de los "estados" correspondientes a un número de caracteres taxonómicos. De esta manera, se podrían tener caracteres relacionados con la ausencia o presencia de alguna condición, como en el siguiente caso: indumento: ausente; presente. En este ejemplo, el carácter o atributo es la presencia de indumento y los estados correspondientes son indumento "ausente" o indumento "presente". A la vez, se podrían tener caracteres que representan condiciones cualitativas, por ejemplo: forma de tricomas: lineales; claviformes; capitados. Debe aclararse que los caracteres son mutuamente exclusivos y aunque pueden combinarse en el caso de presentar dos o más condiciones, no es posible indicar condiciones intermedias, a menos que se definan explícitamente como estados adicionales.

También pueden usarse caracteres cuantitativos, tanto basados en números discretos como en números continuos (el caso de medidas, por ejemplo) según el sistema en uso.

Aunque el formato para la elaboración y presentación de los caracteres es algo diferente para cada uno de los diferentes sistemas que actualmente están siendo aplicados (v.gr. Pankhurst, 1983; Dallwitz, 1980), todos requieren de la preparación de una matriz o tabla básica de datos (Morse, 1974).

En la matriz quedan representados los diferentes taxa (o individuos) y los diferentes caracteres taxonómicos observados en cada taxon. Posteriormente, esa información se transforma o se codifica según las especificaciones del sistema en uso y se transfiere a la computadora para su almacenamiento. La matriz con la información transformada compone la base de datos sobre la cual se efectúan diferentes tareas florísticas. De éstas, la más directa es la descripción; los datos se ordenan automáticamente en posiciones previamente definadas por el usuario al igual que ciertas especificaciones gramaticales y se imprimen. Con esta clase de metodología es posible proseguir directa y automáticamente desde la matriz de datos hasta la producción de originales en tipografía listos para la imprenta, como se ha hecho para los géneros de gramíneas de Australia (Watson y Dallwitz, 1980).

Una aplicación importante de la base de datos es la producción de medios auxiliares para la identificación (para un explicación más detallada ver Pankhurst, 1978; Sneath y Sokal, 1973, y Allkin, 1979). Por ejemplo, por medio de la búsqueda automática de caracteres con estados que dividen los taxa en grupos equitativos (o que permiten una división basada en prioridades previamente definidas) se construye una clave dicotómica convencional a los taxa.

Otro uso es la transferencia de los datos a tarjetas perforadas de computación (Pankhurst, 1978). Cada tarjeta corresponde al estado de un carácter, y las perforaciones a cada uno de los taxa que demuestran el estado. En la práctica, se escoge, en cualquier orden, los caracteres a ser consultados reteniendo las diferentes tarjetas referentes a los estados presentes en el ejemplar desconocido. Se superponen las tarjetas hasta que sólo una perforación queda sin taparse, la cual representa el resultado de la identificación.

Esto forma un tipo de clave de acceso múltiple generalmente llamado policlave. El método es extremadamente útil con material fragmentario que no puede ser identifica- 
do con el uso de una clave dicotómica, porque permite que el usuario escoja los caracteres a usar.

La consulta interactiva es otro método en el que el biólogo elige los caracteres. El sistema de manejo de la base de datos permite que el usuario participe en una "conversación" que se conduce mediante una serie de preguntas y respuestas, llegando a la identificación del ejemplar en cuestión. Además de ser de utilidad con material incompleto, algunos sistemas ayudan al usuario a escoger los mejores caracteres para separar taxa, a buscar caracteres diagnósticos o a comparar taxa.

Una alternativa más es la comparación directa entre las condiciones presentes en el ejemplar desconocido y las condiciones en los taxa representados en la base de datos. El biólogo describe el especimen nuevo utilizando el conjunto de caracteres. Después de transferir la información a la computadora, se calcula automáticamente la semejanza (número de estados en común) entre el desconocido y los contenidos en la matriz.

Este método es de especial interés en el caso de nuevos taxa, ya que evalúa todos los caracteres simultáneamente (un método politético, en contraste con métodos monotéticos, los cuales requieren del uso de un solo carácter a la vez y del acuerdo absoluto con la descripción de cada taxon) y permite de detección de grados de afinidad entre el ejemplar desconocido y los taxa descritos.

Una aplicación de este tipo de datos en México ha sido la preparación de un sistema para la identificación interactiva de las familias de angiospermas en el estado de Veracruz (Gómez-Pompa et al., 1984). Estos métodos, en general, ofrecen opciones de especial aplicabilidad en situaciones donde hay pocos biólogos experimentados para efectuar identificaciones, preparar claves o producir trabajos descriptivos. Obviamente, establecer la base de datos es laborioso, pero después de incorporar la información al sistema, el mismo conjunto de datos puede ser aprovechado para numerosas aplicaciones. A su vez, puede mejorar la eficiencia de trabajos futuros, porque cualquier cambio o adición se refleja automáticamente en todos los productos del sistema.

Al igual que en la mayoría de los sistemas curatoriales, nomenclaturales o bibliográficos, algunos sistemas descriptivos permiten la búsqueda de información específica dentro de la matriz. Por ejemplo, en el Proyecto Vicieae (Bisby et al., 1983) se utiliza el programa EXIR (Abbott, 1976) para la recuperación de información de la base de datos. De esta forma se podría solicitar una lista de los diferentes taxa con flores rojas y presentes en Italia, o utilizar cualquier otro carácter o combinación de caracteres para tener acceso a la información. Asimismo permite escoger los subconjuntos de la información apropiados para las necesidades de algún usuario o grupo de usuarios en particular.

\section{Desventajas y Beneficios}

Sería injusto presentar una introducción a las aplicaciones florísticas de bases de datos sin mencionar algunas de las dificultades que pueden encontrarse durante los procesos de implementación y manejo. Como ha comentado Shetler (1974) los métodos computarizados no son una panacea, ni remplazarán muchas de las labores del taxónomo. Por el contrario, en algunas situaciones pueden dar origen a nuevos problemas de otra índole, relacionados con aspectos organizativos, técnicos o de recursos humanos y físicos. Afortunadamente, con el paso del tiempo y las correspondientes mejorías en 
equipo y metodología estos problemas han ido disminuyendo.

Sin duda uno de los obstáculos más significativos en el uso de métodos automáticos es la dificultad para tener acceso a una computadora. Hace algunos años, esto formaba un impedimento serio en cuanto a la aplicación de métodos para bases de datos. Hoy en día es posible mantenerlos en microcomputadoras de precio moderado, especialmente cuando se complementan con espacio adicional de memoria en la forma de discos adicionales o utilizando uno permanente (el llamado disco duro). Recientemente ha habido una proliferación de dicos permanentes para uso en computadoras de tamaño personal, lo cual representa una forma económica de alcanzar los niveles de computación posibles que hace muy pocos años solamente en las máquinas más grandes se podía alcanzar.

Asimismo, en la actualidad se encuentran varios sistemas de programas disponibles en el mercado para microcomputadora que son apropiados para bases de datos taxonómicos, especialmente de tipo curatorial o bibliográfico. Originalmente diseñados para aplicaciones en el comercio o en la administración, muchos de ellos permiten el almacenamiento de grandes números de registros y ofrecen varias alternativas para la recuperación de la información (Freeston, 1984). Al mismo tiempo, son flexibles y relativamente sencillos de modificar para casos especiales. Las mejores fuentes de información actuales sobre los nuevos sistemas de equipo y de programas son las numerosas revistas semiespecializadas y populares sobre computación.

Es importante reconocer que formar una base inicial de datos descriptivos o capturar todo el contenido de etiquetas de un herbario puede requerir muchas horas de trabajo. A pesar de que el equipo moderno facilita el trabajo de transferir datos escritos a la computadora, el proceso puede ser lento, especialmente durante las etapas iniciales. Sin embargo, utilizar una metodología rigurosa de este tipo puede conducir a un trabajo de investigación disciplinado y ordenado, con resultados confiables que pueden mantenerse siempre al día y presentarse en cualquier formato.

Llevar a cabo la planeación y el esfuerzo necesarios para crear una base de datos puede ofrecer ventajas importantes, ya que eventualmente el tiempo de trabajo invertido en un principio se compensa con el cumplimiento de muchas y diversas tareas. Los beneficios más importantes se resumen a continuación.

1) La posibilidad de revisar, modificar y mantener al corriente todos los datos, lo cual no es factible cuando se almacena la información en las formas tradicionales o cuando se presenta solamente en la forma de publicaciones (que se tienen que reeditar para incorporar nueva información). Una vez recopilada, la información puede estar a disposición de cualquier investigador, quien podrá aprovechar y complementar las labores de recopilación en lugar de repetirlas.

2) La disponibilidad inmediata de la información para uso o revisión durante cualquier etapa del proceso de desarrollo.

3) La recuperación flexible de la información que sólo permite la selección de datos pertinentes. Por ejemplo, con un banco curatorial, si interesan sólo los datos acerca de las fechas de floración de cierta especie, se pueden consultar sin tener que recuperar el resto de la información de cada una de las etiquetas.

4) La presentación ajustable de los datos según las necesidades del momento. En una ocasión tal vez sería necesario dar una lista de todas las especies colectadas en una región geográfica en orden alfabético por especie; en otra, se podría presentar la misma infor- 
mación en un formato diferente, por ejemplo ordenada por colector y número.

En el caso muy específico de México, donde el impulso hacia estudios florísticos es relativamente joven y se encuentra en plena etapa de adquisición y consolidación de la información, podría ser provechoso utilizar medios automáticos desde un principio para su registro y organización. Los acervos bibliográficos y los contenidos de los herbarios todavía no han alcanzado números de ejemplares prohibitivos para la incorporación de, por lo menos, parte de la información en bases de datos. Ahora, cuando más que nunca se reconoce la necesidad de estudiar la flora de México, se debe estar consciente de que existe una alternativa viable y una herramienta metodológica valiosa que pueden ser aplicadas a esa tarea.

AgradeCIMIENTOS. Deseamos expresar nuestro agradecimiento al Dr. Arturo Gómez-Pompa por su apoyo y dirección y al Biól. Sergio Avendaño, quien tuvo la amabilidad de revisar el manuscrito.

\section{LITERATURA CITADA}

AввоTт, L.A., 1976. EXIR User's Manual. University of Colorado.

- F. BISBY, y D. ROGERS, 1985. Taxonomic analysis in biology. Columbia University Press, Nueva York, $336 \mathrm{pp}$.

ALLKIN, R., 1979. The evaluation and selection of plant characteristics for use in computer-aided identification. $\mathrm{Ph}$. D. Thesis. The Polytechnic of Central London.

Bisby, F.A., R.S.J. White, T.D. MacFarlane y M.T. Babac. 1983. The Vicieae Database Project: experimental uses of a monographic taxonomic database for species of vetch and pea. En: J. Felsentein (Ed.), Numerical taxonomy. Springer-Verlag, Berlín. pp. 625-629.

CRovelLO, T.J., 1967. Problems in the use of electronic data processing in biological collections. Taxon 16, 481-494.

, 1972. Computerization of data from the Edward Lee Greene Herbarium (ND-G) at Notre Dame. Brittonia 24:131-141.

y R. MACDONALD, 1970. Index of EDP-IR proyects in systematics. Taxon 19(1): 63-76.

DallwitZ, M.J., 1980. A general system for coding taxonomic descriptions. Taxon 29:41-46.

Forero, E. y F.J. PereirA, 1967. EDP-IR in the National Herbarium of Colombia (COL). Taxon 25:85-94.

FREESTON, M.W., 1984. The implementation of databases on small computers. En: R. Allkin y F. Fisby (eds.), Databases in systematics, Systematics. Association Special. Vol. 26. Academic Press, Londres pp. 43-52.

GibBS Russell, G.E. y P. ConSAlves, 1984. PRECIS. A curatorial and biogeographic system. En: R. Allkin y F. Bisby (eds.), Databases in systematics. Systematic Association Special. Vol. 26. Academic Press, Londres pp. 137-154.

Gómez-Pompa, A., N. Moreno, L. Gama, V. SoSa y R. Allkin, 1984. Flora of Veracruz progress and prospects. En: R. Allkin y F. Bisby (eds.), Databases in sistematics. Systematics Association Special. Vol. 26. Academic Press, Londres. pp. 165-174.

- , N. Moreno, V. SOSA, L. GIDDInGS y M. SOTO, 1985. Flora of Veracruz Project: an update on database management of collections and related information. Taxon 34:645-649.

— C. VÁZQUeZ-YANES y S. GuevarA, 1972. The tropical rain forest; a nonrenewable resource. Science $177: 762-765$.

Heywood, V.H., D.M. Moore, L.N. Derrick, K.A. Mitchell y J. Van Scheepen, 1984. The European taxonomic, floristic and biosystematic documentation system-an introduction. En: R. Allkin y F. Bisby (eds.), Databases in systematics. Systematics Association Special. Vol. 26. Academic Press, Londres, pp. 79-89.

Krauss, H.E., 1973. The information system design for the Flora North America Program. Brittonia 25:119-134.

LOCKHEED INFORMATION Systems (sin fecha). A brief guide to dialog searching. Palo Alto, California. 
LUCAS, G.LL. 1984. Databases in systematics: A summing up. En: R. Allkin y F. Bisby (eds.), Databases in systematics. Systematics Association Special. Vol. 26. Academics Press, Londres. pp. 321-324.

MACDONALD, R.D. 1966. Electronic data processing methods for botanical garden and arboretum records. Taxon 15:291-295.

MASCHERPA, J.M. y G. BOCQUET, 1984. An outline for a database within a major herbarium. En: R. Allkin y F. Bisby (eds.), Databases in systematics. Systematics Association special. Vol. 26. Academic Press, Londres, pp. 235-248.

MorSE, L.E., 1974. Computer-assisted storage and retrieval of the data of taxonomy and systematics. Taxon 23:29-43.

OlverA-FOnSECA, S. y A. Gómez-POMPA, 1973. Ensayo de procesamiento de datos para la flora de Veracruz. En: A. Gómez-Pompa y A. Butanda (eds.), El uso de computadoras en la flora de Veracruz. Universidad Nacional Autónoma de México. México, pp. 42-94.

PAnkhurst, R.J., 1978. Biological identificacion. Edward Arnold, Londres.

, 1983. The construction of a floristic database. Taxon 32(2): 193-202.

- 1984. A review of herbarium catalogues. En: R. Allkin y F. Bisby (eds.), Databases in systematics. Systematics Association Special. Vol. 26 Academic Press, Londres, pp. 155-164.

Prance, G.T., 1977. Floristic inventory of the tropics. Where do we stand? Ann. Missouri Bot. Gard. 64:659-684.

SCHEINVAR, L., A. GÓMEZ-PomPa y L. AlOnSO, 1967. Sistema automático de recuperación de información para el Herbario Nacional del Instituto de Biología de la UNAM. Anales Inst. Biol., UNAM, Ser. Bot. 38(1):203-250.

SHETLER, S.G., 1974. Demythologizing biological data bankin. Taxon 23:71-100.

SNEATH, P. y R. SOKAL. 1973. Numerical taxonomy: the principles and practice of numerical classification. W.H. Freeman, San Francisco.

Soper, J.H. y H. Perring, 1967. Data processing in the herbarium and museum. Taxon 16:13-18.

Systems Development Corporation, 1975. Orbit User Manual. Santa Mónica, California.

Watson, L y M.J. DALWITZ, 1980. Australian grass genera. Anatomy, morphology and keys. Research School of Biological Sciences, Australian National University, Canberra. 